\title{
O Manual de Utilizador de um Software de Análise Qualitativa: as perceções dos utilizadores do webQDA
}

Fábio Freitas ${ }^{1}$, Francislê Neri de Souza ${ }^{2}$, António Pedro Costa ${ }^{3}$ e Sónia Mendes ${ }^{4}$

fabiomauro@ua.pt,fns@ua.pt,pcosta@ludomedia.pt, smendes@ludomedia.pt

${ }^{1}$ Departamento de Educação da Universidade de Aveiro (Centro de Investigação Didática e Tecnologia na Formação de Formadores), 3810-193, Aveiro, Portugal

${ }^{2}$ Departamento de Educação da Universidade de Aveiro (Centro de Investigação Didática e Tecnologia na Formação de Formadores), 3810-193, Aveiro, Portugal

${ }^{3}$ Departamento de Educação da Universidade de Aveiro (Centro de Investigação Didática e Tecnologia na Formação de Formadores), 3810-193, Aveiro, Portugal

${ }^{4}$ Ludomedia, 3720-626, Oliveira de Azeméis, Portugal

DOI: 10.17013/risti.19.107-117

Resumo: Um dos fatores fundamentais no processo de desenvolvimento de um software é o Design de Interação, mais precisamente a Usabilidade, responsável, em grande parte, pela eficiente utilização de uma determinada aplicação. Com esse propósito, designers conceituados como Dieter Rams, vão ainda mais longe, afirmando que o recurso ao bom design deverá tornar um produto tão compreensível, ao ponto de ser autoexplicativo, tornando desnecessário o recurso a Manual de Instruções (Vitsœ, 2016). Será que a aplicação dos princípios de bom design torna os produtos tecnológicos tão intuitivos que dispensam o recurso ao Manual de Utilizador? Este estudo pretende dar a conhecer a perceção dos utilizadores relativamente ao uso do Manual de Utilizador do software de análise qualitativa webQDA ${ }^{\circledR}$, ao nível de frequência com que é consultado, à identificação das operações que levam o utilizador a recorrer ao Manual, em que tipo de suporte é mais explorado, entre outros.

Palavras-chave: Manual de Utilizador; Usabilidade; Software de Análise Qualitativa.

\section{The User Manual of Qualitative DataAnalysis software: the perceptions of webQDA users}

Abstract: One of the key factors in a software development process is the Interaction Design, Usability more precisely, largely responsible for the efficient use of a particular application. For this purpose, renowned designers like Dieter Rams, go even further, stating that the use of good design should make a product as understandable as to be self-explanatory, making the instructions manual unnecessary (Vitsœ, 2016). 
Does the application of good design principles make technology products so intuitive that they do not require the use of the User Manual? This study aims to present the perception of users regarding the use of qualitative analysis software User Guide webQDA®, understanding the level of frequency with which they refer to the manual, identifying operations that lead the users to resort to it, the supports in which the manual is more frequently consulted, among other information..

Keywords: User Manual; Usability; Qualitative Analysis Software.

\section{Introdução}

O recurso a Manuais de Utilizador há muito que faz parte do nosso quotidiano, tendo um papel verdadeiramente relevante no momento em que adquirimos novos produtos para os quais necessitamos de orientações para o seu manuseamento. A tipologia dos Manuais tende a variar conforme o produto, diversificando entre os volumosos livros relativos a software informático, passando pelos comuns cadernos dos produtos industriais e elétricos, pelas discretas brochuras dos dispositivos móveis, até à quase inexistência de Manuais físicos das aplicações para esses mesmos dispositivos. Qualquer que seja o tipo de formato ou de suporte em que existam, os Manuais de utilizador têm a função de ser um elo de comunicação por excelência entre o fabricante de um determinado produto e o consumidor final (Abreu \& Rocha, 2015).

Mas será que nos dias de hoje as pessoas ainda investem parte do seu tempo a ler ou consultar Manuais de Utilizador? Sentirá o utilizador maior predisposição para consultar um manual em papel comparativamente a outros meios? Alguns estudos, como o de Novick \& Ward (2006), afirmam que poucos utilizadores de aplicações informáticas dedicam tempo a consultar os respetivos Manuais, optando por recorrer a outras soluções, tais como, ajuda on-line, solicitação de auxílio a um colega ou experimentando por tentativa e erro. Um dos motivos que parecem explicar essa falta de disposição dos utilizadores para recorrerem aos Manuais poderá justificar-se pelo facto de os utilizadores demonstrarem uma preferência para informações mais processuais ${ }^{1}$ em detrimento das informações não-processuais ${ }^{2}$. Porém, o entendimento de conceitos não-processuais é muitas vezes necessário para um completo entendimento e desempenho de determinadas operações. Nesse sentido, há autores que chamam a atenção para uma necessidade urgente de investigações que encontrem caminhos que contrariem a resistência dos utilizadores às informações não-processuais (van Loggem, Lundin, \& Loggem, 2013).

Já no que se refere às características visuais dos manuais, alguns investigadores confirmam que a utilização de recursos gráficos minimalistas pode oferecer um grande potencial de apoio aos utilizadores (Black, Carroll, \& McGuigan, 1987; Huang, Twidale, Lu, \& Twidale, 2005). Outros autores defendem ainda a ideia de que, devido à falta de compreensibilidade e eficiência de alguns Manuais, a solução pode passar pelo desenvolvimento de Manuais

\footnotetext{
${ }^{1}$ Entenda-se por "informações processuais” todo o tipo de informações que estão relacionadas com as indicações mais práticas e relativas ao "processo" de execução de uma determinada operação.

${ }^{2}$ Entenda-se por "informações não-processuais" todo o tipo de informações que estão relacionadas com as indicações de foro mais teórico, explanativo e metodológico, contextualizando a temática na qual se deseja executar uma determinada operação.
} 
com interface de voz (Alvarez et al., 2010) que, dessa forma, se tornem mais rápidos e eficazes no momento em que é necessário apresentar soluções aos utilizadores. Estes dados encontram apoio em estudos que apontam dificuldades manifestadas por diversos utilizadores em navegar em sistemas de ajuda, especialmente no que diz respeito a encontrar termos de pesquisa úteis, bem como uma generalizada insatisfação ao nível das explicações encontradas (Novick \& Ward, 2006). Por outro lado Atlas (1998) defendia que o método mais simples para desenvolver Manuais Técnicos fáceis de serem usados, seria através do método "User Edit", isto é, levar um utilizador inexperiente a trabalhar numa aplicação onde apenas pode recorrer ao Manual de Utilizador. Os erros e as hesitações manifestados no decorrer do "User Edit" poderiam, então, evidenciar os pontos fracos desse Manual e assim possibilitar o aprimoramento das soluções oferecidas.

Todavia, o facto de alguns utilizadores poderem encontrar e aceder corretamente às informações que procuram, nem sempre é sinónimo de sucesso nas suas tentativas para trabalhar com um determinado software (van Loggem, 2014). Perante estes factos, é imperativo que, em pleno século XXI, exista um sustentado interesse académico que impulsione o recurso a padrões ISO no desenvolvimento de documentação de apoio para os utilizadores de software (van Loggem, 2014). Nesse sentido, este estudo tem como propósito fazer um levantamento das perceções dos utilizadores de um software de análise qualitativa, em relação à utilização do Manual de Utilizador, possibilitando assim o envolvimento dos utilizadores no desenvolvimento de um futuro Manual virtual que possa estar em harmonia com padrões de Usabilidade e de Experiência de Utilizador (Costa, Coelho, Tavares, \& Freitas, 2015)mathematics teaching and learning are subject to an intensive process of innovation and restructuring, not only in terms of content and goals, but also, and especially, in terms of the strategies to implement the teaching of these same contents. On the other hand, the current situation highlights the importance that technological artefacts provide in defining these strategies. In this context, came the idea of developing a platform called WEBMAT-manipulatives, which will consist of a tool where users design their work proposals in the area of mathematics based on manipulatives (eg, logic blocks, cuisenaire rods, geometric blocks.

Devido ao facto de existir uma evidente ambiguidade na terminologia dos Manuais - Manual de Utilizador; Manual de Instruções; Manual Técnico; Guia de Instruções; Guia Prático; Livro de Instruções; etc. - este estudo adotará a nomenclatura "Manual de Utilizador”, pois consideramos que esta designação é mais abrangente no seu conteúdo, não envolvendo apenas as componentes mais processuais e técnicas, mas ampliando o mesmo para uma dimensão mais metodológica.

\section{O webQDA ${ }^{\circledR}$ 2.0 e o seu Manual de Utilizador}

$\mathrm{O}$ webQDA ${ }^{\circledR}$ é um software de apoio à análise qualitativa que funciona num ambiente colaborativo e que poderá ser utilizado quer por estudantes, como por docentes, investigadores/pesquisadores. Este software disponibiliza igualmente ferramentas interativas e de partilha de tarefas, o que possibilita a validação de processos e de produtos, permitindo dessa forma que outros investigadores avaliem à distância, de forma total ou parcial, as definições de "categorias" codificadas e os seus respetivos conteúdos (Neri-de-Souza, Costa, \& Moreira, 2011). 
Outra grande vantagem do webQDA ${ }^{\circledR}$ em relação aos demais softwares de análise qualitativa, prende-se com a sua compatibilidade com diversos sistemas operativos, possibilitando assim o acesso aos projetos em qualquer computador com conexão à Internet, o que acaba por se traduzir em enorme proveito no que concerne à mobilidade, bem como a possibilidade de adicionar e trabalhar com arquivos armazenados na "Cloud".

No presente momento, o webQDA ${ }^{\circledR}$ encontra-se na versão 3.0, versão essa que acrescentou diversos benefícios, tais como: a possibilidade de gerir os projetos em plataformas móveis (tablets e smatphones) e uma linguagem de programação mais estável e amigável para o utilizador. Todavia este estudo debruçou a sua atenção no Manual utilizado na versão 2.0, por ser essa a versão disponível ao público no momento da elaboração deste estudo.

O Manual de Utilizador do webQDA ${ }^{\circledR}$ 2.0 encontrava-se disponível na função de “Ajuda”, presente no ambiente de trabalho (ver figura 1). Ao aceder a esta função, o utilizador era direcionado para um novo separador onde encontrava o manual em suporte digital (PDF). De referir que nunca existiu uma versão oficial do Manual webQDA ${ }^{\circledR}$ em suporte papel, o que não evitou que alguns dos utilizadores que manifestavam preferência pela consulta em papel acabassem por imprimir de forma pessoal o respetivo Manual. $\mathrm{O}$ mesmo era constituído por 84 páginas, dividido em capítulos temáticos e possuía um índice de figuras (Freitas, Souza, \& Costa, 2016)designers conceituados como Dieter Rams, v \uooe3o ainda mais longe, defendendo que a utiliza $\backslash$ uooe $7 \backslash$ uooe3o do bom design dever $\backslash$ uooe1 tornar um produto $t \backslash$ uooe3o compreens $\backslash$ uooedvel, ao ponto de ser autoexplicativo, tornando assim desnecess $\backslash$ uooe1rio o recurso a Manual de Instru uooe $7 \backslash$ uoof5es (Vits \u0153, 2016.

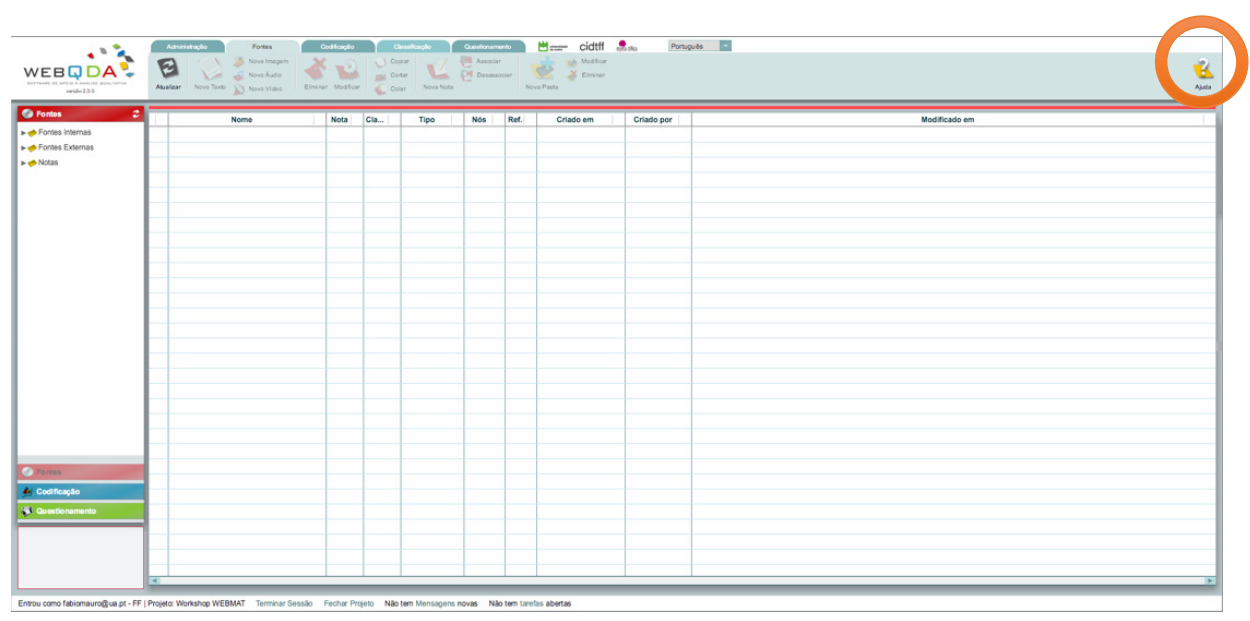

Figura 1 - Acesso ao Manual de Utilizador no ambiente de trabalho do webQDA ${ }^{\circledR}$ 2.0

A atual versão 3.0 do webQDA ${ }^{\circledR}$ disponibiliza um Manual de Utilização Rápida que, contrariamente ao seu antecessor, é fundamentalmente direcionado para as informações processuais, sendo por isso mais conciso (30 páginas). No que se refere à forma de 
consulta deste manual, não existem significativas diferenças em relação à anterior versão, sendo possível aceder ao mesmo através do comando “?” localizado no ambiente de trabalho do webQDA ${ }^{\circledR}$ (ver figura 2).

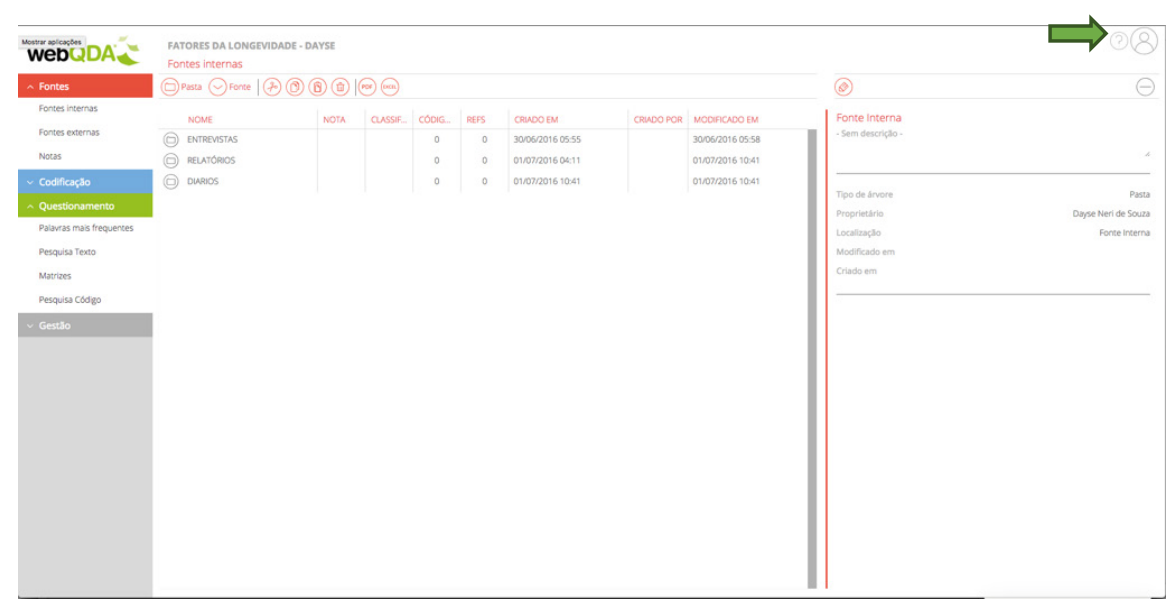

Figura 2 - Acesso ao Manual de Utilizador no ambiente de trabalho do webQDA ${ }^{\circledR}$ 3.0

\section{Metodologia}

Este estudo procurou sondar as perceções dos utilizadores por via do paradigma interpretativo (Coutinho, 2008). O mesmo é fundamentado numa recolha de dados através da aplicação de um inquérito por questionário online disponibilizado a todos os utilizadores do webQDA ${ }^{\circledR}$ via e-mail. Este questionário era composto por 21 questões e estava direcionado para o levantamento das compreensões dos utilizadores do webQDA ${ }^{\circledR}$ em relação ao Manual de Utilizador. O questionário foi dividido em três partes: a primeira parte relacionada com a caracterização dos utilizadores (género, idade, formação académica, profissão e nacionalidade); a segunda parte com o modo como os utilizadores utilizam o Manual de Utilizador e a terceira envolvendo a opinião dos mesmos em relação ao Manual, assim como as mais-valias necessárias para o tornarem mais completo e eficiente na sua utilização. As duas primeiras partes caracterizaram-se pelas questões fechadas, enquanto a terceira parte envolveu as questões abertas e de natureza qualitativa.

Os dados obtidos foram posteriormente analisados com o suporte de dois softwares: o SPSS $^{\circledR}$ (para os dados quantitativos) e o webQDA ${ }^{\circledR}$ (para os dados de natureza qualitativa).

\section{Resultados e Discussão}

O questionário enviado foi respondido por 30 utilizadores do webQDA ${ }^{\circledR}$, sendo que entre eles $57 \%(n=17)$ eram pessoas do sexo feminino e $43 \%(n=13)$ eram pessoas do sexo masculino, tendo idades compreendidas entre os 31 e os 68 anos. A grande maioria dos utilizadores $(70 \%, n=21)$ possuía o grau de doutoramento, havendo $23 \%(n=7)$ que 
estavam a realizar o doutoramento e apenas $1 \%(n=1)$ tinham o grau de mestre. Estes dados aparentam demonstrar que o público-alvo deste software encontra-se essencialmente entre utilizadores com o grau de doutor ou com doutoramento em desenvolvimento. Já no que se refere à atividade exercida por estes utilizadores, a esmagadora maioria (63\%, $\mathrm{n}=19$ ) desempenhava a função de docente e/ou investigador/pesquisador, existindo $13 \%$ $(n=4)$ que exerciam outras atividades e apenas 10\% $(n=3)$ eram estudantes.

No que diz respeito aos anos de utilização do software, os inquiridos revelaram uma grande diversidade de experiências com este aplicativo, sendo que os com 3 anos de experiência $(27 \%, n=8)$ foram aqueles que responderam em maioria ao questionário, seguidos pelos com mais de 5 anos $(20 \%, n=6), 17 \%(n=5)$ revelaram não saber há quanto tempo utilizavam o software, $13 \%(n=4)$ há 2 e 4 anos e $10 \%(n=3)$ há pelo menos 1 ano.

No que concerne à utilização do Manual de Utilizador do webQDA ${ }^{\circledR}$, a grande maioria dos inquiridos $(70 \%, n=21)$ confirmou já ter utilizado, enquanto 30\% $(n=9)$ afirmaram que nunca o tinham feito. Entre aqueles que já haviam consultado o Manual, foi possível verificar que essas consultas se deveram, maioritariamente, a dúvidas relacionadas com operações no Sistema de Questionamento do webQDA ${ }^{\circledR}$ (Matrizes, Matrizes Triangulares, Pesquisa de Código) $(68 \%, n=21)$, seguido das dificuldades em perceber o funcionamento do Sistema de Codificação (Códigos Livres, Códigos em Árvore, Descritores, Classificações/Atributos) $(58 \%, n=18)$. Quando questionados sobre a última vez que tinham sentido necessidade de esclarecer dúvidas durante a utilização do software, 37\% (n=11) afirmaram que sentiram essa necessidade há mais de 12 meses, havendo 20\% $(n=6)$ que confessaram nunca terem sentido qualquer necessidade de esclarecer dúvidas. Os restantes inquiridos dividiramse entre o "há menos de 3 meses" $(13 \%, n=4), 13 \%(n=4)$ entre os 3 e os 6 meses e $13 \%$ $(n=4)$ entre os 6 e os 12 meses. Um facto interessante de constatar está relacionado com a existência de uma maior percentagem de utilizadores do Manual entre as pessoas do sexo masculino $(85 \%, \mathrm{n}=11)$, comparativamente com as pessoas do sexo feminino $(59 \%, \mathrm{n}=10)$.

No que respeita ao recurso preferencial dos utilizadores, em caso de dúvidas durante a utilização do webQDA ${ }^{\circledR}$ (ver figura 3), é possível constatar que o Manual de Utilizador é aquele que colhe maior número de referências (17), seguido do recurso a "Outro utilizador" mais experiente com 13 referências. Estes dados parecem contrariar o que foi defendido por Novick \& Ward (2006) em relação ao facto de poucos utilizadores de aplicações informáticas utilizarem Manuais de Utilizador. Estes dados podem darnos alguma luz sobre a compreensão da relevância que o Manual de Utilizador tem no processo de apoio aos utilizadores, bem como na forma como ele demonstra ser o recurso por excelência nos momentos de dúvidas durante a utilização do software.
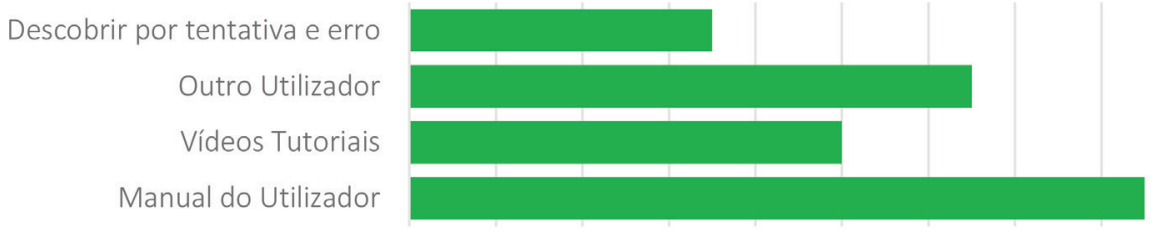

$\begin{array}{llllllllll}0 & 2 & 4 & 6 & 8 & 10 & 12 & 14 & 16 & 18\end{array}$

Figura 3 - Número de referências relativas ao recurso preferencial dos utilizadores, em caso de dúvidas, durante a utilização do webQDA ${ }^{\circledR}$ 
Quando inquiridos em relação ao suporte no qual utilizam o Manual de Utilizador, 83\% $(n=25)$ referem que utilizam o Manual em formato digital (PDF), havendo 10\% ( $n=3)$ que recorre ao suporte em papel. Estes dados poderiam não ter grande significado, não fosse o caso de o Manual de Utilizador do webQDA ${ }^{\circledR}$ apenas existir em formato digital, o que demonstra que os utilizadores acabam por imprimir, por sua própria iniciativa, o Manual disponibilizado em PDF. Ainda questionados sobre a relevância de um Manual de Utilizador em papel, 63\% (n=19) manifestaram a importância da existência do manual nesse suporte, enquanto $37 \%(\mathrm{n}=11)$ demonstraram ser totalmente irrelevante. Estes dados parecem confirmar uma antiga ideia de que, apesar dos grandes desenvolvimentos tecnológicos, os Manuais de Utilizador no futuro iriam continuar a privilegiar o uso do papel (McKee, 1985). Todavia, há estudos (Novick \& Ward, 2006) que declararam que, apesar do reconhecimento da importância e utilidade deste suporte, poucos são os utilizadores que têm Manuais dos aplicativos informáticos que utilizam em papel.

Quando os utilizadores foram inquiridos em relação aos atributos que não existiam na versão do Manual 2.0, mas que poderiam revelar-se mais-valias para um futuro Manual de Utilizador do webQDA, a opção pela inclusão de vídeos demonstrativos foi a que granjeou maior preferência $(77 \%, n=24)$, enquanto a opção de busca por temas foi mencionada por $65 \%(n=20)$. Estes dados parecem demonstrar uma maior apetência dos utilizadores para recursos que possibilitem uma maior agilidade na busca das informações que procuram, bem como uma tendência para indicações mais exemplificativas e visuais em detrimento das teóricas.

As questões abertas apresentadas no questionário tinham como principal objetivo sondar a opinião dos utilizadores em relação às características ${ }^{3}$ que mais apreciavam no manual de utilizador (ver tabela 1) e às características que menos apreciavam (ver tabela 2). De forma a estruturar as apreciações dos utilizadores, criou-se duas dimensões nas quais podemos compor o Manual de Utilizador: uma dimensão referente aos conteúdos escritos e visuais (vertente técnica e metodológica) e outra dimensão relacionada com a Experiência de Utilizador (relacionada com o lado emotivo ocasionado pela consulta do Manual) (ISO, 2010). Estas duas dimensões, por sua vez, foram subdivididas em temáticas, temáticas essas referidas pelos utilizadores no questionário e agrupadas nestas duas grandes dimensões. Na tabela 1 é possível verificar que na dimensão "Conteúdo Escrito/Visual" os inquiridos referiram situações relacionadas com: "Acessibilidade Textual” (a forma clara e simples como as orientações do Manual estavam apresentadas); "Grafismos e Imagens" (referente a tudo o que está relacionado com ilustrações explicativas e design gráfico); "Objetividade” (alusiva à forma concisa e direta com que o texto do Manual foi elaborado) e a "Organização" (respeitante à disposição e organização dos temas e indicações do Manual). Por outro lado, na dimensão "Experiência de Utilizador", os inqueridos mencionaram dados relativos à "Usabilidade" (eficácia e eficiência na consulta do Manual) e "Acessibilidade Instrucional” (relativa à boa orientação das instruções).

Perante os dados recolhidos foi possível então constatar que, no que toca ao "Conteúdo Escrito/Visual”, os utilizadores referiram que a "Acessibilidade Textual” e os "Grafismos/

\footnotetext{
${ }^{3}$ Entende-se por “características” todos os elementos/funcionalidades patenteadas no Manual e que podem influenciar a compreensão do utilizador em relação aos processos para os quais pretende adquirir informações.
} 
Imagens" são os elementos que mais se destacam no Manual de Utilizador do webQDA ${ }^{\circledR}$. Contudo, a dimensão da "Experiência de Utilizador" foi aquela que os utilizadores mais referiram, centrando as suas opiniões na "Acessibilidade Instrucional”:

“Clareza, a facilidade na sua utilização, abrangência das orientações;" Utilizador 2

“(...) me permita ver cómo se aplica lo que se ha explicado teoricamente e que pueda ir con facilidad al tema que me interese sin dar continuas vueltas por el manual(...)” - Utilizador 8

\begin{tabular}{l|l|l|l|l|l}
\hline \multicolumn{3}{c|}{ Conteúdo Escrito/Visual } & \multicolumn{2}{c}{ Experiência de Utilizador } \\
\hline $\begin{array}{l}\text { Acessibilidade } \\
\text { Textual }\end{array}$ & $\begin{array}{l}\text { Grafismos/ } \\
\text { Imagens }\end{array}$ & Objetividade & Organização & Usabilidade & $\begin{array}{l}\text { Acessibilidade } \\
\text { Instrucional }\end{array}$ \\
\hline 9 & 8 & 3 & 3 & 2 & 13 \\
\hline
\end{tabular}

Tabela 1 - Referências às características que os utilizadores mais apreciam no Manual webQDA 2.0

Estes dados parecem indiciar uma eficiente relação dos utilizadores com o Manual de Utilizador, além de transparecer que o mesmo cumpre com os seus objetivos instrucionais.

Na tabela 2, referente às características que os utilizadores menos apreciam no Manual de Utilizador, temos as mesmas duas grandes dimensões, porém com algumas características novasreferidaspelosutilizadoresnadimensão “ConteúdoEscrito/Visual”.Entreelasencontrase: a "Densidade Textual" (relativa à forma maciça como as indicações estão descritas); "Pouco detalhe" (referente a processos operacionais pouco detalhados); "FAQ" (espaço para perguntas frequentes) e "Não Existir Suporte em Papel” (relacionado com o suporte físico do Manual). Um dado que sobressai nesta tabela está relacionado com a diminuição do número de referências de cariz negativo relativas ao Manual, comparativamente com as de cariz positivo. Contudo, os utilizadores inquiridos não deixaram de referir que a característica "Grafismos/Imagens" foi aquela que maior insatisfação lhes proporcionou. Este maior consenso deve-se em muito à pouca qualidade (ao nível da resolução) das imagens, sendo esse um dos pontos mais referenciados pelos utilizadores:

“a pouca resolução das imagens "print screen" - Utilizador 1

“A qualidade das imagens (desfocadas).” - Utilizador 3

“A fraca qualidade e resolução das figuras demonstrativas” - Utilizador 6

Porém, as observações negativas ao nível dos “Grafismos/Imagens” não se restringiram unicamente à pouca qualidade das figuras demonstrativas. Outros utilizadores referiram, por exemplo, a existência de "poucos esquemas" (Utilizador 7). Esta apreciação parece complementar as opiniões apresentadas por outros utilizadores, que declararam que o Manual apresentava uma grande "Densidade Textual”:

"Longo, demasiado texto em algumas secções” - Utilizador 12

"Muito texto e poucos esquemas" - Utilizador 12

“(...) é denso na sua informação” - Utilizador 30 
Estes dados aparentam indicar que o Manual de Utilizador, apesar de ser denso na sua informação teórica, no que se refere às indicações visuais esquemáticas das operações a serem realizadas, é mais limitado e não cumpre com eficiência o seu objetivo. Parece ficar manifestado que alguns utilizadores do webQDA dão maior preferência às informações processuais (com recurso a figuras com esquemas processuais) em detrimento das informações não-processuais.

É curioso verificar que, apesar destas opiniões críticas em relação à "Densidade Textual” do Manual de Utilizador, existem outros inquiridos que manifestam precisamente a opinião contrária ao referirem que:

\section{“Alguns passos de funções não estão detalhados." - Utilizador 2 \\ “Ausência de explicações de alguns itens.” - Utilizador 26}

Estas opiniões aparentemente contrastantes demonstram a singularidade de cada utilizador, evidenciando a necessidade premente de os Manuais de Utilizador poderem vir a ser personalizáveis na sua utilização, adaptando-se assim ao perfil de cada utilizador. Todavia, as mesmas parecem, uma vez mais, reforçar a ideia de que a quantidade não é sinónimo de eficiência na informação prestada. Apesar de o Manual ser completo em conteúdos teóricos e metodológicos, no que se refere às informações passo-a-passo (informações processuais) ainda há melhoramentos a serem feitos.

\begin{tabular}{lcccccc}
\hline \multicolumn{4}{c}{ Conteúdo Escrito/Visual } & & \multicolumn{2}{c}{ Experiência de Utilizador } \\
\hline $\begin{array}{l}\text { Densidade } \\
\text { Textual }\end{array}$ & $\begin{array}{c}\text { Grafismos/ } \\
\text { Imagens }\end{array}$ & $\begin{array}{c}\text { Pouco } \\
\text { Detalhe }\end{array}$ & FAQ & $\begin{array}{c}\text { Não existir } \\
\text { suporte em papel }\end{array}$ & Usabilidade & $\begin{array}{c}\text { Acessibilidade } \\
\text { Instrucional }\end{array}$ \\
\hline 3 & 6 & 2 & 1 & 1 & 1 & 3 \\
\hline
\end{tabular}

Tabela 2 - Referências às características que os utilizadores menos apreciam no Manual webQDA 2.0

Outro dado relevante de referir na tabela 2, está relacionado com a dimensão da "Experiência de Utilizador", pois esta foi a que menos referências negativas reuniu. Esse facto torna-se ainda mais relevante no sentido em que confirma a satisfação dos utilizadores ao nível da Experiência de Utilizador (ver tabela 1). Este dado parece uma vez mais atestar que, ao nível da Experiência de Utilizador, o atual Manual de Utilizador cumpre com as suas funções e proporciona uma eficaz consulta.

\section{Considerações Finais}

Quando não existe um eficiente sistema de ferramentas de aprendizagem autónoma para os utilizadores, pode-se correr o risco de sobrecarregar as empresas de software com pedidos de suporte. Tal situação poderia traduzir-se num aumento de trabalho para os elementos do serviço de apoio, assim como aumentar a insatisfação dos utilizadores pelo tempo que terão de esperar até obterem a resposta de que necessitam (Serbest, Goksen, Dogan, \& Tokdemir, 2015). Nesse sentido, este estudo pretendeu recolher uma série de dados que possibilitasse uma eficaz compreensão das perceções dos utilizadores do webQDA ${ }^{\circledR}$ em relação ao seu Manual de Utilizador, com o objetivo de aprimorar as soluções de autoaprendizagem disponibilizadas pela aplicação. Assim, os dados recolhidos e parte 
deles aqui patenteados, parecem apontar para a generalizada satisfação dos utilizadores, porém, há uma clara consciência de que a amostra dos inquiridos é ainda muito residual, tendo em conta o número total de utilizadores do webQDA ${ }^{\circledR}$.

Este estudo possibilitou igualmente desmistificar a ideia de que os utilizadores nunca recorrem aos Manuais de Utilizador, bem como demonstrar a relevância de versões dos mesmos em suporte papel.

Esta recolha e análise de perceções propõe ser o início de uma série de estudos que têm como objetivo o desenvolvimento de um protótipo de Manual de Utilizador Virtual para o webQDA ${ }^{\circledR}$, baseado nos princípios de usabilidade e no modelo $4 \mathrm{C}$ (Costa, Reis, \& Loureiro, 2014), sendo nosso objetivo, num próximo estudo, avaliar a Usabilidade e a Experiência de Utilizador do software, de forma a compreender como os utilizadores interagem com o webQDA ${ }^{\circledR}$.

\section{Agradecimentos.}

Gostaríamos de agradecer à Fundação para a Ciência e Tecnologia (FCT) pelo apoio financeiro concedido na realização deste estudo e ao webQDA, através da empresa Micro IO e da empresa Ludomedia, pela disponibilidade para a recolha de dados para sustentar esta investigação.

\section{Referências}

Abreu, A., \& Rocha, Á. (2015). The electronic booklet in the school-Family relationship: Perceptions of teachers and guardians. In 2015 10th Iberian Conference on Information Systems and Technologies (CISTI) (pp. 1-10). IEEE.

Alvarez, I., Martin, A., Dunbar, J., Taiber, J., Wilson, D.-M., \& Gilbert, J. E. (2010). Voice interfaced vehicle user help. In Proceedings of the 2nd International Conference on Automotive User Interfaces and Interactive Vehicular Applications - AutomotiveUI '1O (p. 42). New York, New York, USA: ACM Press. http://doi.org/10.1145/1969773.1969782

Atlas, M. (1998). The user edit: Making Manuals Easier to Use. ACM SIGDOC Asterisk Journal of Computer Documentation, 22(3), 5-6. http://doi. org/10.1145/381808.381811

Black, J. B., Carroll, J. M., \& McGuigan, S. M. (1987). What kind of minimal instruction manual is the most effective. ACM SIGCHI Bulletin, 18(4), 159-162. http://doi.org/10.1145/1165387.275623

Costa, A. P., Coelho, E. B., Tavares, L. C., \& Freitas, F. (2015). Envolvimento dos Utilizadores na definição de Requisitos: o caso da plataforma WEBMAT. RISTI - Revista Ibérica de Sistemas e Tecnologias de Informação, (E4), 92-106. http://doi.org/10.17013/risti.e4.92-106

Costa, A. P., Reis, L. P., \& Loureiro, M. J. (2014). Desenvolvimento de Software Educativo : A Coordenação como Fator Crítico de Sucesso. RISTI - Revista Ibérica de Sistemas e Tecnologias de Informação, E2(9), 1-18. http://doi.org/10.17013/ risti.e2.1-18 
Coutinho, C. P. (2008). A qualidade da investigação educativa de natureza qualitativa: questões relativas à fidelidade e validade. Educação Unisinos, 12(1), 5-15. Retrieved from http://repositorium.sdum.uminho.pt/bitstream/1822/7884/1/o05a015_ ARTo1_Coutinho\%5Brev_OK\%5D.pdf

Freitas, F., Souza, F. N. de, \& Costa, A. P. (2016). Manual do Utilizador vs Utilizador do Manual: o caso do webQDA. In Atas CIAIQ2016 - Investigação Qualitativa em Engenharia e Tecnologia (Vol. 4). Porto: Ludomedia.

Huang, J., Twidale, M. B. B., Lu, B., \& Twidale, M. B. B. (2005). Graphical Abstract Help. In Proceedings of the 6th ACM SIGCHI New Zealand chapter's international conference on Computer-human interaction (pp. 83-89). New York, New York, USA: ACM Press. http://doi.org/10.1145/1294211.1294248

ISO. (2010). Ergonomics of human-system interaction - Part 210: Human-centred design for interactive systems. International Standards Organisation (First edit). Geneva.

McKee, J. B. (1985). Computer user manuals in print: Do they have a future? In Proceedings of the 4th annual international conference on Systems documentation - SIGDOC '85 (pp. 8-14). New York, New York, USA: ACM Press. http://doi.org/10.1145/10563.10565

Neri-de-Souza, F., Costa, A. P., \& Moreira, A. (2011). Análise de Dados Qualitativos Suportada pelo Software WebQDA. In Atas da VII Conferência Internacional de TIC na Educação: Perspetivas de Inovação (pp. 49-56). Braga: CHALLANGES2011. Retrieved from https://www.webqda.com/analise-de-dados-qualitativossuportada-pelo-software-webqda/

Novick, D. G., \& Ward, K. (2006). Why Don't People Read the Manual? In Proceedings of the 24th annual conference on Design of communication - SIGDOC 'O6 (p. 11). http://doi.org/10.1145/1166324.1166329

Serbest, S., Goksen, Y., Dogan, O., \& Tokdemir, A. (2015). Design and Implementation of Help Desk System on the Effective Focus of Information System. In Procedia Economics and Finance (Vol. 33, pp. 461-467). Kavala: Elsevier Ltd. http://doi.org/10.1016/S2212-5671(15)01729-3

van Loggem, B. (2014). Software Documentation: a Standard for the 21st Century. In Proceedings of the International Conference on Information Systems and Design of Communication - ISDOC '14 (pp. 149-154). New York, New York, USA: ACM Press. http://doi.org/10.1145/2618168.2618192

van Loggem, B., Lundin, J., \& Loggem, B. Van. (2013). Interaction with User Documentation : A Preliminary Study. In Proceedings of the 2013 International Conference on Information Systems and Design of Communication (pp. 41-46). New York, New York, USA: ACM Press. http://doi.org/10.1145/2503859.2503866

Vitsœ. (2016). Dieter Rams: ten principles for good design. Retrieved from https://www.vitsoe.com/eu/about/good-design 